\title{
Metallographische Mitteilungen aus dem Institut für physikalische Chemie der Universität Göttingen. \\ LXVIII. \\ Über Silicium-Silberlegierungen.
}

Von

G. Arrivaut.

Mit 1 Figur im Text.

Schon im Jahre 1858 hatte WöHLER, ${ }^{1}$ indem er auf Silbernitrat $\mathrm{SiH}_{4}$ einwirken liefs, einen schwarzen Stoff erhalten, welchen er für ein mit Silber gemischtes Silbersilicid hielt. Später, im Jahre 1889 , konstatierte $\mathrm{W}_{\text {ARREN }}{ }^{2}$ dafs Silicium und Silber, ,aufser im status nascendi, wenig verwandt sind". Er präparierte Reguli von Silbersilicid, indem er eine Mischung von kieselfluorwasserstoffsaurem Kalium mit Natrium und Silber zur Rotglut brachte.

Auch de Chatmot ${ }^{3}$ glaubte, indem er eine Mischung von Sand, Kohlenstoff, Kalk und Stücken Silber im elektrischen Strome schmolz, ein krystallisiertes Silbersilicid, das hart genug war, um Glas zu ritzen, erbalten zu haben; denn durch Behandeln dieses Körpers mit verdünnter Salpetersäure konnte er nur einen gewissen Teil des Silbers auflösen und aufserdem hatte sich $\mathrm{SiO}_{2}$ gebildet. Entgegen diesen Annabmen behauptet MoIssan, ${ }^{4}$ dals Silicium und Silber sich nicht verbinden. Bei seinen Versuchen über die Löslichkeit des Siliciums in Silber kam in der Tat kein Silicid zum Vorschein. ${ }^{5}$

Kürzlich stellte nun E. VIaodroux,${ }^{6}$ als er sich mit der Ein-

1 Martins und Wöhler, Ann. Chim. Phys. [3] 54 (1858), 218.

2 Warren, Chem. News 60 (1889), 5; 67 (1896), 303.

3 De Chalmot, Amer. Chem. Journ. 18 (1896), 95.

* Morssan, Compt. rend. 121 (1895), 625.

5 Morssan und Slemens, Compt. rend. 138 (1904), 657 u. 1299.

${ }^{6}$ E. Vigojnoux, Compt. rend. 144 (1907), 1214. 
wirkung des Silbers auf Siliciumtetrachlorid befalste, Reduktionserscheinungen fest, aber auch er erhielt keine Verbindung.

Obgleich es nach dem oben Gesagten sehr wahrscheinlich ist, dafs das Silicium und Silber bei den uns zu Gebote stehenden Temperaturen und experimentellen Bedingungen keine Neigung haben, sich zu verbinden, so könnte man doch einwenden, dals entweder die bei hohen Temperaturen gebildete Verbindung beim Abkühlen wieder zerfiele und so wieder freies Silicium gäbe, das aus Silber krystallisierte, oder dals sie bei dem Versuche, sie auf chemischem Wege zu isolieren, zerfiele. Es erschien mir also nötig, die Frage noch einmal einer systematischen Untersuchung zu unterziehen, was ich auf Anregung von Herrn Prof. Tammann mittels der Methode der ,thermischen Analyse“" auszuführen versuchte.

Das zur Herstellung der Schmelzen benutzte Silber war sehr rein. Das Silicium, welches ich zuerst verwandte, enthielt nur ungefähr $95 \%$ reines Silicium, der Rest bestand hauptsächlich aus Aluminium und Eisen. Um die erhaltenen Resultate zu kontrollieren, verwendete ich jedoch dann im weiteren Verlaufe meiner Untersuchungen ein aus Aluminium umkrystallisiertes Silicium, das ich mit grofser Sorgfalt reinigte. Es enthielt $99 \%$ reines Silicium.

Die erhaltenen Resultate der thermischen Untersuchung sind in der Tabelle 1 zusammengestellt und in Fig. 1 graphisch veranschaulicht.

Bei den Legierungen bis $z u 20 \% \mathrm{Si}$ betrug die angewandte Menge $20 \mathrm{~g}$, später wurden bei den siliciumreicheren Legierungen geringere Mengen genommen, damit das Volumen nicht zu grols würde und die Abkühlungsbedingungen ähnlich blieben.

Um die eutektischen Haltezeiten vergleichen zu können, wurden sie alle auf gleiches Gewicht umgerechnet.

Wie man sieht, besteht die Schmelzkurve aus zwei Kurvenästen, welche bei den Schmelzpunkten des Silbers und Siliciums beginnen und sich bei einer Temperatur von ca. $830^{\circ}$ schneiden in einem Punkte, der einer Konzentration von $4-5 \%$ Silicium entspricht.

Man kann also aus diesem Diagramm ersehen, dafs Silicium und Silber keine Verbindung miteinander geben. Im flüssigen $\mathrm{Zu}$ stande sind sie unbegrenzt mischbar, über die Mischbarkeit im festen Zustande ist folgendes zu sagen: Die Zeitdauer der eutektischen Krystallisation wird auf der Seite des Silbers erst bei 100\% Silber Null, bei $2.5 \%$ zugesetztem Siliciums war auf der Abküh-

Z. anorg. Chem. Bd. 60 . 
Tabelle 1.

\begin{tabular}{|c|c|c|c|c|}
\hline $\begin{array}{c}\mathrm{Si} \\
\text { in } \%\end{array}$ & $\begin{array}{c}\text { Temp. der } \\
\text { prim. Ausscheidg. } \\
\text { in }{ }^{0}\end{array}$ & $\begin{array}{c}\text { Eutektisehe } \\
\text { Temp. } \\
\text { in }\end{array}$ & $\begin{array}{l}\text { Gewicht der } \\
\text { Legierungen } \\
\text { in } g\end{array}$ & $\begin{array}{l}\text { Eutekt. Haltep. } \\
\text { in Sek. } \\
\text { für } 20 \mathrm{~g}\end{array}$ \\
\hline 0 & 950 & & & \\
\hline 2 & 905 & 830 & 20 & 90 \\
\hline 4 & 870 & 830 & 20 & 140 \\
\hline 5 & 940 & 830 & 20 & 160 \\
\hline 7 & 1035 & 830 & 20 & 120 \\
\hline 10 & 1130 & 825 & 20 & 100 \\
\hline 20 & 1255 & 820 & 20 & 90 \\
\hline 30 & 1290 & 820 & 18 & 85 \\
\hline 40 & 1320 & 820 & 16 & 75 \\
\hline 50 & 1340 & 825 & 14 & 57 \\
\hline 60 & 1350 & 820 & 13 & 46 \\
\hline 70 & 1365 & 820 & 12 & 33 \\
\hline 80 & 1380 & 830 & 11 & 20 \\
\hline 90 & 1395 & - & 11 & - \\
\hline 95 & 1405 & - & 10 & - \\
\hline 98 & 1410 & - & 10 & - \\
\hline 100 & 1415 & - & 10 & - \\
\hline
\end{tabular}

lungskurve noch deutlich ein Haltepunkt von 90 Sekunden bei der Temperatur des eutektischen Punktes zu beobachten. Auf der Seite des Siliciums hingegen scheint diese Zeitdauer bei einer Konzentration von $90 \%$ Silicium Null zu werden. Legierungen von 95 und $98 \%$ Silicium, deren Abkühlungskurven aufgenommen wurden, um diese Schlüsse zu bestätigen, gaben bei $830^{\circ}$ keinen Haltepunkt auf der Abkühlungskurve.

Zwischen 90 und $100 \%$ Silicium bilden sich also Mischkrystalle, die bei $90 \%$ Silicium gesättigt sind.

Die mikroskopische Untersuchung der Struktur der Reguli bestätigt dies. Die Legierungen, welche weniger als $4 \%$ Silicium enthalten, weisen keine grofsen Krystalle von primär ausgeschiedenem Silicium auf und bestehen aus Silber und Eutektikum. Bei der Konzentration von $4 \%$ Silicium sieht man bereits einige gesättigte Mischkrystalle, obgleich man sich noch, wie aus dem Diagramme ersichtlich, diesseits des eutektischen Punktes befindet. Da aber diese Krystalle sich nur in dem oberen Teile des Regulus finden und die Dichten der beiden Metalle sehr verschieden sind, so erklärt sich diese Erscheinung möglicherweise aus nicht ganz 
vollständiger Durchmischung, wodurch im oberen Teile der Siliciumgehalt gröfser wäre.

In dem Mafse, wie der Siliciumgehalt wächst, nimmt auch die Menge der Mischkrystalle zu; gleichzeitig nimmt die Menge des

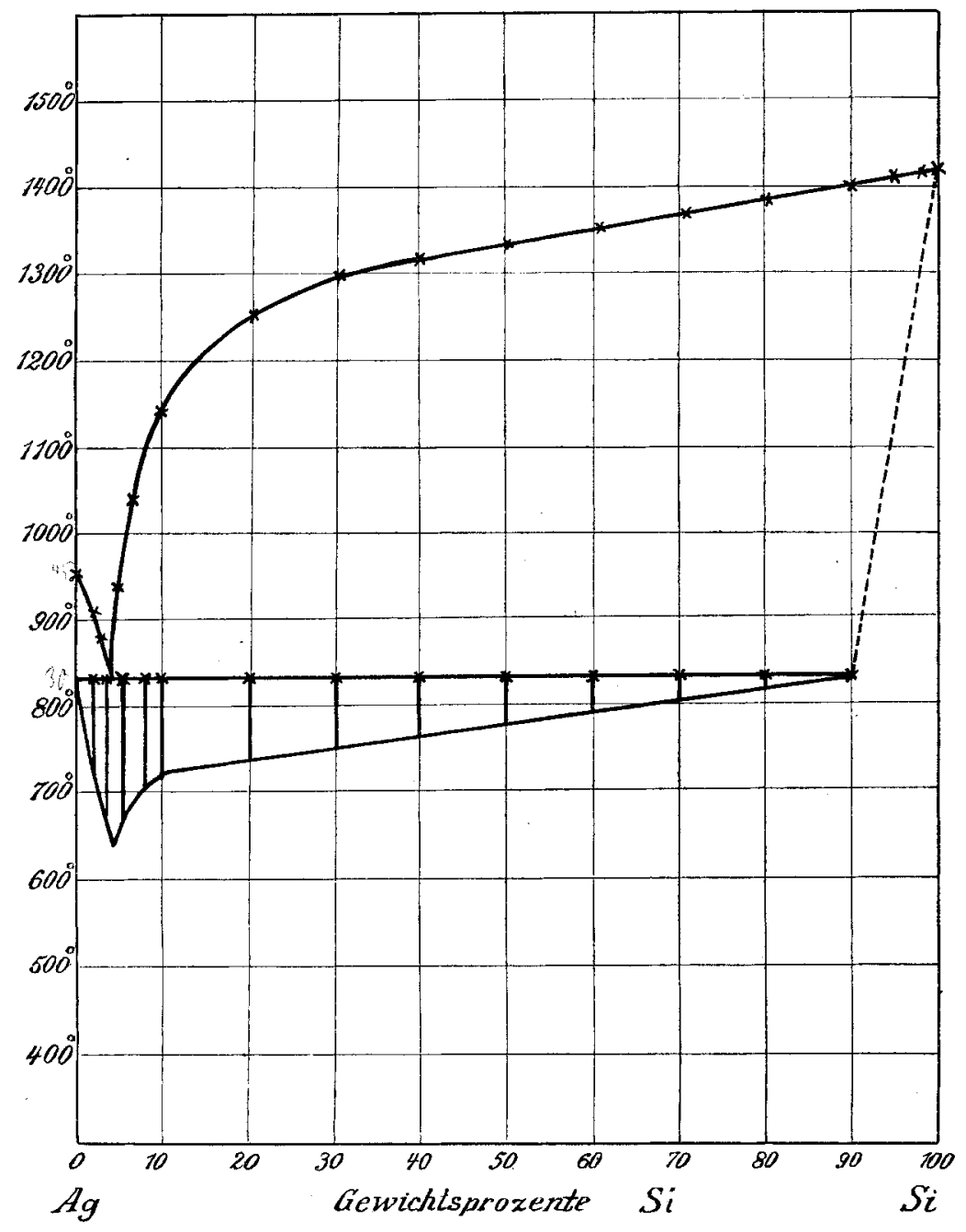

Fig. 1.

Eutektikums ab, um bei. $90 \%$ Silicium ganz zu verschwinden. Von hier an zeigen die Legierungen homogene Struktur.

Man kann also sagen, dals sich bei einer Konzentration von $90 \%$ Silicium gesättigte Mischkrystalle ausscheiden. 
Bei den siliciumreichen Schmelzen beobachtete ich auf dem Regulus eine kleine blanke Perle, die sich deutlich von der grau. blauen Oberfläche abhob. Diese Perle sieht man auch bei reinem Silicium, jedoch ist sie hier glänzend schwarz. Man nimmt diese Erscheinung auch wahr beim Erstarren von Wismut; auch ist sie analog der Beobachtung, die FRÄNKEL ${ }^{1}$ bei den Silicium-Aluminiumlegierungen machte, und spricht ebenfalls für die Annahme, dafs Silicium unter Ausdehnung krystallisiert. Wenn die Schmelze noch Silber enthält, so wird der Teil, der am längsten flüssig ist, das ist das Eutektikum, nach oben gedrückt und bildet die helle Perle. Um diese Tatsachen zu bestätigen, habe ich eine solche Perle analysiert. Ihr Gewicht betrug $0.5505 \mathrm{~g}$ und ich fand:

\begin{tabular}{rr} 
Silber & 94.85 \\
Silicium $\quad 4.90$ \\
\hline 99.75
\end{tabular}

Diese Zahlen entsprechen der Zusammensetzung des Eutektikums, die sich aus dem Diagramm ergibt.

Zum Schlusse sei es mir gestattet, Herrn Prof. Tammand für seine freundliche Anregung und Hilfe bei der Arbeit meinen herzlichsten Dank auszusprechen.

1 W. Fränkel, Z. anorg. Chem. 58 (1908), 154.

Göttingen, Institut für physikalische Chemie.

Bei der Redaktion eingegangen am 15. Oktober 1908. 